\title{
Distributed Synchronization of Stochastic Complex Networks with Time-Varying Delays via Randomly Occurring Control
}

\author{
Xiaoyan Liu, Xiuliang Qiu \\ Chengyi University College, Jimei University, Xiamen, China \\ Email: liuxiaoyan@jmu.edu.cn, qiuxiulian@jmu.edu.cn
}

How to cite this paper: Liu, X.Y. and Qiu, X.L. (2021) Distributed Synchronization of Stochastic Complex Networks with TimeVarying Delays via Randomly Occurring Control. Applied Mathematics, 12, 803-817. https://doi.org/10.4236/am.2021.129054

Received: August 29, 2021

Accepted: September 24, 2021

Published: September 27, 2021

Copyright ( 2021 by author(s) and Scientific Research Publishing Inc. This work is licensed under the Creative Commons Attribution International License (CC BY 4.0).

http://creativecommons.org/licenses/by/4.0/

\section{(c) (i) Open Access}

\begin{abstract}
This paper studies the distributed synchronization control problem of a class of stochastic dynamical systems with time-varying delays and random noise via randomly occurring control. The activation of the distributed adaptive controller and the update of the control gain designed in this paper all happen randomly. Based on the Lyapunov stability theory, LaSalle invariance principle, combined with the use of the properties of the matrix Kronecker product, stochastic differential equation theory and other related tools, by constructing the appropriate Lyapunov functional, the criterion for the distributed synchronization of this type of stochastic complex networks in mean square is obtained.
\end{abstract}

\section{Keywords}

Stochastic Dynamical System, Time-Varying Delay, Distributed

Synchronization, Randomly Occurring Control

\section{Introduction}

In the human society and the objective world in which human society lives, complex networks can be seen everywhere, and there are various complex networks developed and constructed by humans intentionally or unintentionally: interpersonal networks, road networks, railway networks, power networks, the Internet, and news dissemination networks; there are also various non-human complex networks that exist in the objective world: ecological networks, biological neural networks, metabolic networks, animal and plant gene networks, and so on. Due to the rapid development of human science and technology, the rapid development of global integration, and the huge investment in various public 
infrastructures from all over the world, the construction and cognition of various complex networks have achieved unprecedented rapid development. Although this rapid development has greatly improved people's work efficiency and quality of life, such as the popularization of mobile communication networks, social software and GPS positioning and navigation. However, this also brings a variety of new or potential risks to people, such as infectious diseases relying on the rapid spread of complex networks, large-scale power outages caused by power grid failures, and huge losses caused by computer viruses relying on the rapid spread of high-speed networks, etc. Taking into account the various existing and potential important applications, in order to make complex networks better serve humans and eliminate related network risks, the theory and applications of complex networks have gradually become very popular in scientific research in the past few decades. In the field of research, its research has very important application value and academic significance.

Synchronization [1] refers to a phenomenon in which multiple individuals or systems interact in a certain way. Although the initial states of the individuals are different, as the individual states gradually evolve, they eventually tend to be consistent. Synchronization is an important attribute of complex dynamical networks. It is widely present in the real world, such as the unison singing of a large number of crickets, the synchronized flashing of a large number of fireflies that are often seen in the wild on summer nights, the spread of computer viruses, and the synchronized reproduction of periodic cicadas, etc., all of these are wellknown synchronization phenomena. As people discover more and more synchronization phenomena and many potential applications, such as satellite navigation systems, image processing [2] [3], network communication security [4], power network system design, etc. Therefore, synchronization has gradually aroused the interest of researchers from the fields of mathematics, physics, sociology, and engineering, and has been extensively studied in various fields of science and engineering. Studying the synchronization phenomenon of complex networks and the conditions of synchronization has high application significance and theoretical value.

Random phenomenon is a common phenomenon in nature. Many practical systems are inevitably affected by it. For example, signals in complex dynamic networks are often affected by network bandwidth, conductive media, measurement noise, etc., when they are transmitted between nodes in the network. The influence of random factors causes information to be randomly lost or incomplete [5] [6] [7]. Due to the ubiquity of random interference factors, the control and design of the system becomes complicated and difficult. The conventional deterministic model is far from satisfying the requirements. Therefore, it is necessary taking into account the random interference factors inside or outside the system, a stochastic complex network model can be established so as to better and more accurately reflect the inherent essential characteristics of natural and engineering systems. With the in-depth study of complex networks, stochastic dynamic behavior has attracted widespread attention from scholars in various 
fields. In recent years, some important research results in deterministic complex networks have been extended to the research field of stochastic complex networks, and rich results have been obtained, for example, in the reference [8], D. Hunt, G. Korniss, and B Szymanski et al. studied the synchronization of stochastic complex networks with coupled time delays; Z. Wang, Y. Wang, and Y. Liu [9] studied global synchronization for discrete-time stochastic complex networks with randomly occurred nonlinearities and mixed time-delays. Reference [10] studied the synchronization of complex dynamical networks with random coupling time-varying delays and nonlinear coupling terms. Up to now, researchers have proposed many different types of synchronization under different control frameworks, such as: adaptive synchronization [11], distributed synchronization [12] [13], synchronization under impulsive control [14], inphase and antiphase synchronization [15], finite-time synchronization [14] [16], synchronization under periodic intermittent adaptive control [17], synchronization under pinning control [18], synchronization under adaptive pinning control [18], etc. Literature [12] and [13] designed a distributed adaptive controller, the main feature of which is that nodes can more effectively use the local information of their neighbors without the need for global information on the entire network. Considering that the signals in the network system in practical applications may have data packet loss and random failures during the transmission process [19] [20], it takes random disturbances into account, and a Bernoulli stochastic variable is used to simulate this random disturbance, a distributed control that occurs randomly is proposed to simulate packet loss. In the application of actual engineering systems, randomly occurring controller is also worthy of in-depth study, especially the distributed synchronization of stochastic complex networks under randomly occurring control, which has important research value.

In summary, complex network systems will inevitably be affected by random factors such as so-called time-delay and external disturbance. Common delay factors that cause time-delay include: transmission, calculation, communication, and driving; common external disturbance factors include environmental noise, internal random failures and external attacks, etc. In some cases, the stability of the system may be greatly affected in these scenarios, or even completely deviate from the previous plan. Therefore, when considering the synchronization of complex networks, it is necessary to add the above-mentioned transmission delay and other random factors such as time-delay and environmental noise to the model, and design a more robust controller to suppress the negative effects of time-delay and noise to obtain better control accuracy. Although the reference [12] considers that the complex network is disturbed by random mutations, it proposes a method of randomly occurring control and studies the synchronization behavior of stochastic dynamical systems, but it does not take into account the influence of coupling delay and random disturbance term time-delay. The model has certain limitations.

Based on the above point of view, this article generalizes the complex network model proposed in [12], and proposes a more general stochastic dynamical net- 
work model with time-varying delays and environmental noise under randomly occurring control. The model has the following characteristics: we use two Bernoulli random variables to describe the occurrence of distributed adaptive control, and update it according to a certain probability, and study the synchronization stability of stochastic complex networks by considering randomly occurring control and updating law. This paper mainly adopts Lyapunov stability theory and LaSalle invariance principle to study and explore synchronization control problems, and analyze the corresponding network synchronization characteristics. The results of this paper will enrich the synchronization control theory of stochastic dynamical systems on complex networks to a certain extent, and have important theoretical and application value.

\section{Model Description and Preliminaries}

Notations: Let $\mathbb{R}\left(\mathbb{R}^{+}\right)$denote the set of real (positive) numbers. $\mathbb{R}^{n}$ and $\mathbb{R}^{n \times m}$ denote, respectively, the $n$-dimensional Euclidean space and the set of all $n \times m$-dimensional real matrices.

$A>0$ denotes that the matrix $A$ is a symmetric and positive definite matrix. The notation $A^{\mathrm{T}}$ is the transpose of a vector or matrix $A$. I represents the identity matrix with appropriate dimensions. $\|A\|$ denotes the Euclidean norm of a matrix $A$ and $\lambda_{\max }(A)$ (respectively, $\left.\lambda_{\min }(A)\right)$ denotes the maximum (respectively, minimum) eigenvalue of matrix $A$. The symbol trace $(A)$ represents the trace of square matrix $A=\left(a_{i j}\right)_{n \times n}$, i.e. $\operatorname{trace}(A)=\sum_{i=1}^{n} a_{i i} . \otimes$ stands for the Kronecker product. And $E\{X\}$ represents the expectätion of the random variable $X$. Define a graph by $\mathscr{G}=[\vartheta, E]$, where $\vartheta=\{1, \cdots, N\}$ denotes the vertex set and $\mathscr{E}=\{e(i, j)\}$ denotes the edge set. $N(i)$ denotes the neighborhood of vertex $i$ in the sense $N(i)=\{j \in \mathscr{V}: e(i, j) \in \mathscr{E}\}$. In this paper, graph $\mathscr{G}$ is supposed to be undirected $[e(i, j) \in \mathscr{E}$ implies $e(j, i) \in \mathscr{E}]$ and simple (without self-loops and multiple edges). Let $L=\left[l_{i j}\right]_{i, j=1}^{N}$ be the Laplacian matrix of graph $\mathscr{G}$, which is defined as follows: for any pair $i \neq j, l_{i j}=l_{j i}=-1$ if $e(i, j) \in \mathcal{E}$ and $l_{i j}=l_{j i}=0$ otherwise. $l_{i i}=-\sum_{j=1, j \neq i}^{N} l_{i j}$ stands for the degree of vertex $i(i=1,2, \cdots, N)$. Let $(\Omega, \mathcal{F}, \mathcal{P})$ be a complete probability space, where $\Omega$ represents a sample space, $\mathcal{F}$ is called a $\sigma$-algebra, and $\mathcal{P}$ is a probability measure.

In this paper, we consider the following model of a complex network stochastic system with time-varying delays, which can be expressed as:

$$
\begin{aligned}
\mathrm{d} x_{i}(t) \\
=\left\{A x_{i}(t)+f\left(t, x_{i}(t), x_{i}(t-\tau(t))\right)+c \sum_{j \in N(i)} \Gamma\left[x_{j}(t-\tau(t))-x_{i}(t-\tau(t))\right]\right\} \mathrm{d} t \\
+\sigma\left(t, x_{i}(t), x_{i}(t-\tau(t))\right) \mathrm{d} w(t), i=1,2, \cdots, N
\end{aligned}
$$

where $x_{i}(t)=\left[x_{i 1}(t), x_{i 2}(t), \cdots, x_{i n}(t)\right]^{\mathrm{T}} \in \mathbb{R}^{n}(i=1,2, \cdots, N)$ is the state vector of the $i$ th vertex, $A \in \mathbb{R}^{n \times n}$ is a constant matrix,

$f\left(t, x_{i}(t), x_{i}(t-\tau(t))\right): \mathbb{R} \times \mathbb{R}^{n} \times \mathbb{R}^{n} \rightarrow \mathbb{R}^{n}$ is a continuous nonlinear vector-va- 
lued function. The positive constant $c>0$ is the coupling strength of the network. The inner coupling matrix $\Gamma=\operatorname{diag}\left\{\eta_{1}, \eta_{2}, \cdots, \eta_{n}\right\}>0$ is a constant diagonal matrix. $\tau(t)$ is a time-varying delay. Furthermore, $\sigma\left(t, x_{i}(t), x_{i}(t-\tau(t))\right): \mathbb{R} \times \mathbb{R}^{n} \times \mathbb{R}^{n} \rightarrow \mathbb{R}^{n}$ is the noise intensity function vector, and $w(t)$ is a scalar Brownian motion defined on $(\Omega, \mathcal{F}, \mathcal{P})$ satisfying $E\{\mathrm{~d} w(t)\}=0$ and $E\left\{[\mathrm{~d} w(t)]^{2}\right\}=\mathrm{d} t$.

According to the definition and properties of the above Laplacian matrix $L=\left[l_{i j}\right]_{i, j=1}^{N}$, it is easy to know that Formula (1) can be rewritten as

$$
\begin{aligned}
\mathrm{d} x_{i}(t)= & \left\{A x_{i}(t)+f\left(t, x_{i}(t), x_{i}(t-\tau(t))\right)-c \sum_{j=1}^{N} l_{i j} \Gamma x_{j}(t-\tau(t))\right\} \mathrm{d} t \\
& +\sigma\left(t, x_{i}(t), x_{i}(t-\tau(t))\right) \mathrm{d} w(t), i=1,2, \cdots, N
\end{aligned}
$$

Additionally, From the Gershgorin disk theorem, all the eigenvalues of the Laplacian matrix $L$ corresponding to graph $\mathscr{G}$ satisfy $0=\lambda_{1}(L) \leq \lambda_{2}(L) \leq \cdots \leq \lambda_{N}(L)$. furthermore, $\mathscr{G}$ is connected if and only if $\lambda_{2}(L)>0$ : i.e., $L$ is irreducible.

In order to achieve the synchronization of the stochastic complex network in (1) or (2), controllers are added to each vertex.

$$
\begin{aligned}
& \mathrm{d} x_{i}(t)=\left\{A x_{i}(t)+f\left(t, x_{i}(t), x_{i}(t-\tau(t))\right)+c \sum_{j \in N(i)} \Gamma\left[x_{j}(t-\tau(t))\right.\right. \\
& \left.\left.-x_{i}(t-\tau(t))\right]+u_{i}(t)\right\} \mathrm{d} t+\sigma\left(t, x_{i}(t), x_{i}(t-\tau(t))\right) \mathrm{d} w(t), i=1,2, \cdots, N
\end{aligned}
$$

where $u_{i}(t)$ is a distributed adaptive controller.

For the $i$ th vertex, $u_{i}(t)$ is designed as

$$
u_{i}(t)=\rho(t) \sum_{j \in N(i)} \varepsilon_{i}(t) \Gamma\left(x_{j}(t)-x_{i}(t)\right), i=1,2, \cdots, N
$$

where $\varepsilon_{i}(t)$ is the control strength of vertex $i$.

In (4), $\rho(t)$ is a Bernoulli stochastic variable that describes the following random events for (3):

$$
\left\{\begin{array}{l}
\text { Event 1:(3) experiences (4) } \\
\text { Event } 2:(3) \text { does not experience (4) }
\end{array}\right.
$$

Let $\rho(t)$ be defined by

$$
\rho(t)= \begin{cases}1, & \text { if Event } 1 \text { occurs } \\ 0, & \text { if Event } 2 \text { occurs }\end{cases}
$$

where the probability of event $\{\rho(t)=1\}$ is $\operatorname{Pr}\{\rho(t)=1\}=\rho \in[0,1]$, so the expectation of random variable $\rho(t)$ is $E\{\rho(t)\}=\rho$.

The distributed controller (4) in this paper takes stochastic disturbances into account and uses a Bernoulli stochastic variable to simulate this random disturbance. The distributed controller $u_{i}(t)$ occurs in a probabilistic manner and uses feedback information from neighboring points. Different from the conventional adaptive controller, the distributed controller $u_{i}(t)$ is not always im- 
plemented and it can model control failure in a stochastic way. In short, randomly occurring distributed control can effectively use the information of neighboring points to simulate real-world disturbances.

$\varepsilon_{i}(t)$ in (4) is updated according to the following randomly occurring distributed updating law:

$$
\mathrm{d} \varepsilon_{i}(t)=\xi(t) \alpha\left[\sum_{j \in N(i)}\left(x_{j}(t)-x_{i}(t)\right)\right]^{\mathrm{T}} \Gamma\left[\sum_{j \in N(i)}\left(x_{j}(t)-x_{i}(t)\right)\right] \mathrm{d} t, i=1,2, \cdots, N(7)
$$

where $\alpha>0$ and $\xi(t)$ is a Bernoulli stochastic variable representing the following random events for (7):

$$
\left\{\begin{array}{l}
\text { Event } 3: \varepsilon_{i}(t) \text { experiences }(7) \\
\text { Event } 4: \varepsilon_{i}(t) \text { does not experience }(7)
\end{array}\right.
$$

Similarly, Let $\xi(t)$ be defined by

$$
\xi(t)= \begin{cases}1, & \text { if Event } 3 \text { occurs } \\ 0, & \text { if Event } 4 \text { occurs }\end{cases}
$$

where the probability of event $\{\xi(t)=1\}$ is $\operatorname{Pr}\{\xi(t)=1\}=\xi \in[0,1]$, so the expectation of stochastic variable $\xi(t)$ is $E\{\xi(t)\}=\xi$.

Remark 1: If $\rho=1$ and $\xi=1$, the control and updating rule will be simplified to normal control and updating law. If $\rho=0$ and $\xi=0$, the problem considered in this article will be simplified to the synchronization of complex networks without controllers.

It can be seen from the above model that the complex network studied in this paper has the following characteristics:

1) The model contains random terms that characterize environmental noise.

2) The activation of the controller and the updating law of control gain both occur in a probabilistic manner, and the distributed synchronization of stochastic complex networks is studied by considering the random occurrence of control and update laws.

3) Considering the effect of time-delays, and the time-delays are time-varying, the model is more general.

The following definition, assumptions and lemmas are needed for deriving the main results.

Definition 1 [12]: Let $x_{i}(t)(1 \leq i \leq N)$ be the solution of the stochastic complex network with time-varying delays in (1) or (3), if they satisfy the following condition:

$$
\lim _{t \rightarrow \infty} E \sum_{i=1}^{N}\left\|x_{i}(t)-x_{j}(t)\right\|^{2}=0, i, j=1,2, \cdots, N
$$

then the stochastic complex network is said to achieve synchronization in mean square.

Lemma 1 (Itô formula) [21]: Let $x(t)$ be an Itô process on $t \geq 0$ with the stochastic differential

$$
\mathrm{d} x(t)=f(t) \mathrm{d} t+g(t) \mathrm{d} w(t)
$$


Let $V(x, t)$ be a real-valued function, which is continuously twice differentiable in $x$ and once differentiable in $t$. Then $V(x(t), t)$ is again an Itô process with the stochastic differential given by

$$
\begin{aligned}
& \mathrm{d} V(x(t), t) \\
& =\left[V_{t}(x(t), t)+V_{x}(x(t), t) f(t)+\frac{1}{2} \operatorname{trace}\left(g^{\mathrm{T}}(t) V_{x x}(x(t), t) g(t)\right)\right] \mathrm{d} t \\
& \quad+V_{x}(x(t), t) g(t) \mathrm{d} w(t)
\end{aligned}
$$

Lemma 2 [22]: For any $x, y \in \mathbb{R}^{n}$ and any positive definite symmetric matrix $P \in \mathbb{R}^{n \times n}$, the following inequalities hold

$$
\pm 2 x^{\mathrm{T}} y \leq x^{\mathrm{T}} P x+y^{\mathrm{T}} P y .
$$

Lemma 3 [23]: Assuming that $P \in \mathbb{R}^{n \times n}$ is a positive definite matrix, for any $x \in \mathbb{R}^{n}$, the following inequalities hold

$$
\lambda_{\text {min }}(P)\|x\|^{2} \leq x^{\mathrm{T}} P x \leq \lambda_{\max }(P)\|x\|^{2} .
$$

Assumption 1 [24] [25]: Assume that there exist constants $\beta_{1} \geq 0$ and $\beta_{2} \geq 0$, such that the nonlinear function $f(\cdot, \cdot, \cdot): \mathbb{R} \times \mathbb{R}^{n} \times \mathbb{R}^{n} \rightarrow \mathbb{R}^{n}$ in the system (1) satisfy

$$
\begin{aligned}
& \left\|f\left(t, \xi_{1}(t), \xi_{1}(t-\tau(t))\right)-f\left(t, \xi_{2}(t), \xi_{2}(t-\tau(t))\right)\right\|^{2} \\
& \leq \beta_{1}\left\|\xi_{1}(t)-\xi_{2}(t)\right\|^{2}+\beta_{2}\left\|\xi_{1}(t-\tau(t))-\xi_{2}(t-\tau(t))\right\|^{2}
\end{aligned}
$$

Which holds for all $\xi_{1}(t), \xi_{2}(t) \in \mathbb{R}^{n}$ and $t>0$.

Assumption 2 [26]: Assume that there exist constants $\gamma_{1}>0$ and $\gamma_{2}>0$, such that the nonlinear function $\sigma(\cdot, \cdot, \cdot): \mathbb{R} \times \mathbb{R}^{n} \times \mathbb{R}^{n} \rightarrow \mathbb{R}^{n}$ in the system (1) satisfy

$$
\begin{aligned}
& \operatorname{trace}\left[\left(\sigma\left(t, \xi_{1}, \eta_{1}\right)-\sigma\left(t, \xi_{2}, \eta_{2}\right)\right)^{\mathrm{T}}\left(\sigma\left(t, \xi_{1}, \eta_{1}\right)-\sigma\left(t, \xi_{2}, \eta_{2}\right)\right)\right] \\
& \leq \gamma_{1}\left(\xi_{1}-\xi_{2}\right)^{\mathrm{T}}\left(\xi_{1}-\xi_{2}\right)+\gamma_{2}\left(\eta_{1}-\eta_{2}\right)^{\mathrm{T}}\left(\eta_{1}-\eta_{2}\right)
\end{aligned}
$$

Which holds for all $\xi_{1}(t), \xi_{2}(t), \eta_{1}(t), \eta_{2}(t) \in \mathbb{R}^{n}$ and $t>0$.

Assumption 3: The time-varying delay $\tau(t)$ is a bounded continuous differentiable function, which satisfies: $0 \leq \dot{\tau}(t) \leq \bar{\tau}<1$. where $\dot{\tau}(t)$ represents the derivative of $\tau(t)$ to $t$.

\section{Main Results}

In this section, we will derive the main results of the distributed synchronous control of a class of stochastic dynamical systems (3) with time-varying delays and random noise via randomly occurring control and updating law.

Theorem 1: Suppose that the nonlinear function $f(\cdot, \cdot, \cdot)$ in the stochastic complex network (3) satisfies Assumption 1, the noise intensity function $\sigma(\cdot,,, \cdot)$ satisfies Assumption 2, and the time-varying delays $\tau(t)$ satisfies Assumption 3 , then the stochastic complex network (3) will achieve synchronization in mean square under the distributed adaptive controller (4) and updating 
law (7), if

$$
\begin{aligned}
& {\left[\frac{1}{2}\left(\beta_{1}+\beta_{2}+\gamma_{1}+\gamma_{2}+1\right)+\sqrt{\lambda_{\max }\left(A^{\mathrm{T}} A\right)}\right] I_{N}} \\
& <\left[\rho b \eta-\frac{c}{2}-P \bar{\tau}-\frac{c}{2} \lambda_{\max }\left(\Gamma \Gamma^{\mathrm{T}}\right)\right] L
\end{aligned}
$$

Proof: Let $e_{i j}(t)=x_{i}(t)-x_{j}(t), \forall i, j=1,2, \cdots, N$ and $x=\left[x_{1}^{\mathrm{T}}, x_{2}^{\mathrm{T}}, \cdots, x_{N}^{\mathrm{T}}\right]^{\mathrm{T}} \in \mathbb{R}^{n N}$.

Consider the following Lyapunov function

$$
V(t)=V_{1}(t)+V_{2}(t)+V_{3}(t)
$$

where

$$
\begin{gathered}
V_{1}(t)=\frac{1}{4} \sum_{i=1}^{N} \sum_{j \in N(i)} e_{i j}^{\mathrm{T}} e_{i j} \\
V_{2}(t)=\frac{\rho}{2 \xi \alpha} \sum_{i=1}^{N}\left(\varepsilon_{i}(t)-b\right)^{2} \\
V_{3}(t)=\sum_{i=1}^{N} \int_{t-\tau(t)}^{t}\left[\sum_{j \in N(i)} e_{i j}(s)\right]^{\mathrm{T}} P\left[\sum_{j \in N(i)} e_{i j}(s)\right] \mathrm{d} s
\end{gathered}
$$

where $b$ is a positive constant.

According to (3) and (4) we can easily obtain

$$
\begin{aligned}
& \mathrm{d} e_{i j}(t)=\mathrm{d} x_{i}(t)-\mathrm{d} x_{j}(t) \\
&=\left\{A\left[x_{i}(t)-x_{j}(t)\right]+\left[f\left(t, x_{i}(t), x_{i}(t-\tau(t))\right)-f\left(t, x_{j}(t), x_{j}(t-\tau(t))\right)\right]\right. \\
&+c \sum_{k \in N(i)} \Gamma\left[x_{k}(t-\tau(t))-x_{i}(t-\tau(t))\right]-c \sum_{m \in N(j)} \Gamma\left[x_{m}(t-\tau(t))-x_{j}(t-\tau(t))\right] \\
&\left.+\rho(t) \sum_{k \in N(i)} \varepsilon_{i}(t) \Gamma\left[x_{k}(t)-x_{i}(t)\right]-\rho(t) \sum_{m \in N(j)} \varepsilon_{j}(t) \Gamma\left[x_{m}(t)-x_{j}(t)\right]\right\} \mathrm{d} t \\
&+\left[\sigma\left(t, x_{i}(t), x_{i}(t-\tau(t))\right)-\sigma\left(t, x_{j}(t), x_{j}(t-\tau(t))\right)\right] \mathrm{d} w(t) \\
&=\left\{A e_{i j}(t)+\tilde{f}\left(t, e_{i j}(t), e_{i j}(t-\tau(t))\right)-2 c \sum_{j \in N(i)} \Gamma e_{i j}(t-\tau(t))\right. \\
&\left.-2 \rho(t) \varepsilon_{i}(t) \Gamma \sum_{j \in N(i)} e_{i j}(t)\right\} \mathrm{d} t+\tilde{\sigma}\left(t, e_{i j}(t), e_{i j}(t-\tau(t))\right) \mathrm{d} w(t)
\end{aligned}
$$

where

$$
\begin{aligned}
& \tilde{f}\left(t, e_{i j}(t), e_{i j}(t-\tau(t))\right)=f\left(t, x_{i}(t), x_{i}(t-\tau(t))\right)-f\left(t, x_{j}(t), x_{j}(t-\tau(t))\right) \\
& \tilde{\sigma}\left(t, e_{i j}(t), e_{i j}(t-\tau(t))\right)=\sigma\left(t, x_{i}(t), x_{i}(t-\tau(t))\right)-\sigma\left(t, x_{j}(t), x_{j}(t-\tau(t))\right)
\end{aligned}
$$

By the Lemma 1 (Itô formula), the stochastic derivative of $V(t)$ can be obtained as

$$
\mathrm{d} V(t)=\mathscr{L} V(t) \mathrm{d} t+\frac{1}{2} \sum_{i=1}^{N} \sum_{j \in N(i)} e_{i j}^{\mathrm{T}} \tilde{\sigma}\left(t, e_{i j}(t), e_{i j}(t-\tau(t))\right) \mathrm{d} w(t)
$$


and according to (21), the Itô differential operator $\mathscr{L}$ is given as

$$
\begin{aligned}
& \mathscr{L} V(t)=\frac{1}{2} \sum_{i=1}^{N} \sum_{j \in N(i)} e_{i j}^{\mathrm{T}}(t)\left\{A e_{i j}(t)+\tilde{f}\left(t, e_{i j}(t), e_{i j}(t-\tau(t))\right)\right. \\
& \left.-2 c \sum_{j \in N(i)} \Gamma e_{i j}(t-\tau(t))-2 \rho(t) \varepsilon_{i}(t) \Gamma \sum_{j \in N(i)} e_{i j}\right\} \\
& +\sum_{i=1}^{N} \frac{\rho}{\xi} \varepsilon_{i}(t) \cdot \xi(t)\left[\sum_{j \in N(i)} e_{i j}\right]^{\mathrm{T}} \Gamma\left[\sum_{j \in N(i)} e_{i j}\right] \\
& -\sum_{i=1}^{N} \frac{\rho}{\xi} b \cdot \xi(t)\left[\sum_{j \in N(i)} e_{i j}\right]^{\mathrm{T}} \Gamma\left[\sum_{j \in N(i)} e_{i j}\right]+\sum_{i=1}^{N}\left[\sum_{j \in N(i)} e_{i j}(t)\right]^{\mathrm{T}} P\left[\sum_{j \in N(i)} e_{i j}(t)\right] \\
& -\sum_{i=1}^{N}\left[\sum_{j \in N(i)} e_{i j}(t-\tau(t))\right]^{\mathrm{T}} P\left[\sum_{j \in N(i)} e_{i j}(t-\tau(t))\right] \cdot(1-\dot{\tau}(t)) \\
& +\frac{1}{4} \sum_{i=1}^{N} \sum_{j \in N(i)} \tilde{\sigma}^{\mathrm{T}}\left(t, e_{i j}(t), e_{i j}(t-\tau(t))\right) \tilde{\sigma}\left(t, e_{i j}(t), e_{i j}(t-\tau(t))\right) \\
& =\frac{1}{2} \sum_{i=1}^{N} \sum_{j \in N(i)} e_{i j}^{\mathrm{T}}(t) A e_{i j}(t)+\frac{1}{2} \sum_{i=1}^{N} \sum_{j \in N(i)} e_{i j}^{\mathrm{T}}(t) \tilde{f}\left(t, e_{i j}(t), e_{i j}(t-\tau(t))\right) \\
& -c \sum_{i=1}^{N}\left[\sum_{j \in N(i)} e_{i j}(t)\right]^{T} \Gamma\left[\sum_{j \in N(i)} e_{i j}(t-\tau(t))\right] \\
& -\sum_{i=1}^{N}\left[\sum_{j \in N(i)} e_{i j}(t)\right]^{T} \rho(t) \varepsilon_{i}(t) \Gamma\left[\sum_{j \in N(i)} e_{i j}(t)\right] \\
& +\sum_{i=1}^{N} \frac{\rho}{\xi} \varepsilon_{i}(t) \cdot \xi(t)\left[\sum_{j \in N(i)} e_{i j}\right]^{\mathrm{T}} \Gamma\left[\sum_{j \in N(i)} e_{i j}\right] \\
& -\sum_{i=1}^{N} \frac{\rho}{\xi} b \cdot \xi(t)\left[\sum_{j \in N(i)} e_{i j}\right]^{\mathrm{T}} \Gamma\left[\sum_{j \in N(i)} e_{i j}\right] \\
& +\sum_{i=1}^{N}\left[\sum_{j \in N(i)} e_{i j}(t)\right]^{\mathrm{T}} P\left[\sum_{j \in N(i)} e_{i j}(t)\right] \\
& -\sum_{i=1}^{N}\left[\sum_{j \in N(i)} e_{i j}(t-\tau(t))\right]^{\mathrm{T}} P\left[\sum_{j \in N(i)} e_{i j}(t-\tau(t))\right] \cdot(1-\dot{\tau}(t)) \\
& +\frac{1}{4} \sum_{i=1}^{N} \sum_{j \in N(i)} \tilde{\sigma}^{\mathrm{T}}\left(t, e_{i j}(t), e_{i j}(t-\tau(t))\right) \tilde{\sigma}\left(t, e_{i j}(t), e_{i j}(t-\tau(t))\right)
\end{aligned}
$$

Taking expectations of $\rho(t)$ and $\xi(t)$, we obtain $E[\rho(t)]=\rho$ and $E\left[\frac{\rho}{\xi} \xi(t)\right]=\rho$, furthermore

$$
\begin{aligned}
E \mathscr{L} V(t)= & E\left\{\frac{1}{2} \sum_{i=1}^{N} \sum_{j \in N(i)} e_{i j}^{\mathrm{T}}(t) A e_{i j}(t)+\frac{1}{2} \sum_{i=1}^{N} \sum_{j \in N(i)} e_{i j}^{\mathrm{T}}(t) \tilde{f}\left(t, e_{i j}(t), e_{i j}(t-\tau(t))\right)\right. \\
& -c \sum_{i=1}^{N}\left[\sum_{j \in N(i)} e_{i j}(t)\right]^{\mathrm{T}} \Gamma\left[\sum_{j \in N(i)} e_{i j}(t-\tau(t))\right]
\end{aligned}
$$




$$
\begin{aligned}
& -\rho b \sum_{i=1}^{N}\left[\sum_{j \in N(i)} e_{i j}\right]^{\mathrm{T}} \Gamma\left[\sum_{j \in N(i)} e_{i j}\right]+\sum_{i=1}^{N}\left[\sum_{j \in N(i)} e_{i j}(t)\right]^{\mathrm{T}} P\left[\sum_{j \in N(i)} e_{i j}(t)\right] \\
& -\sum_{i=1}^{N}\left[\sum_{j \in N(i)} e_{i j}(t-\tau(t))\right]^{\mathrm{T}} P\left[\sum_{j \in N(i)} e_{i j}(t-\tau(t))\right] \cdot(1-\dot{\tau}(t)) \\
& \left.+\frac{1}{4} \sum_{i=1}^{N} \sum_{j \in N(i)} \tilde{\sigma}^{\mathrm{T}}\left(t, e_{i j}(t), e_{i j}(t-\tau(t))\right) \tilde{\sigma}\left(t, e_{i j}(t), e_{i j}(t-\tau(t))\right)\right\}
\end{aligned}
$$

From the definitions of $e_{i j}, x$ and the Laplacian matrix $L$, we get

$$
\begin{gathered}
\frac{1}{2} \sum_{i=1}^{N} \sum_{j \in N(i)} e_{i j}^{\mathrm{T}} e_{i j}=x^{\mathrm{T}}\left(L \otimes I_{n}\right) x \\
\sum_{i=1}^{N}\left[\sum_{j \in N(i)} e_{i j}\right]^{\mathrm{T}} \Gamma\left[\sum_{j \in N(i)} e_{i j}\right]=x^{\mathrm{T}}\left(L^{2} \otimes \Gamma\right) x
\end{gathered}
$$

Then, using Lemma 3 and (25), it's not hard to get

$$
\begin{aligned}
\frac{1}{2} \sum_{i=1}^{N} \sum_{j \in N(i)} e_{i j}^{\mathrm{T}}(t) A e_{i j}(t) & \leq \sqrt{\lambda_{\max }\left(A^{\mathrm{T}} A\right)} \cdot \frac{1}{2} \sum_{i=1}^{N} \sum_{j \in N(i)} e_{i j}^{\mathrm{T}} e_{i j} \\
& =\sqrt{\lambda_{\max }\left(A^{\mathrm{T}} A\right)} \cdot x^{\mathrm{T}}\left(L \otimes I_{n}\right) x
\end{aligned}
$$

By Lemma 2 and 3, Assumptions 1, (25), and (26), the following inequalities can be obtained:

$$
\begin{aligned}
& \frac{1}{2} \sum_{i=1}^{N} \sum_{j \in N(i)} e_{i j}^{\mathrm{T}}(t) \tilde{f}\left(t, e_{i j}(t), e_{i j}(t-\tau(t))\right) \\
& \leq \frac{1}{2}\left(\beta_{1}+1\right) \cdot \frac{1}{2} \sum_{i=1}^{N} \sum_{j \in N(i)} e_{i j}^{\mathrm{T}}(t) e_{i j}(t)+\frac{1}{2} \beta_{2} \cdot \frac{1}{2} \sum_{i=1}^{N} \sum_{j \in N(i)} e_{i j}^{\mathrm{T}}(t-\tau(t)) e_{i j}(t-\tau(t)) \\
& \leq \frac{1}{2}\left(\beta_{1}+1\right) \cdot x^{\mathrm{T}}\left(L \otimes I_{n}\right) x+\frac{1}{2} \beta_{2} \cdot x^{\mathrm{T}}(t-\tau(t))\left(L \otimes I_{n}\right) x(t-\tau(t))
\end{aligned}
$$

and

$$
\begin{aligned}
- & c \sum_{i=1}^{N}\left[\sum_{j \in N(i)} e_{i j}\right]^{\mathrm{T}} \Gamma\left[\sum_{j \in N(i)} e_{i j}(t-\tau(t))\right] \\
\leq & \frac{c}{2} \lambda_{\max }\left(\Gamma \Gamma^{\mathrm{T}}\right) \sum_{i=1}^{N}\left[\sum_{j \in N(i)} e_{i j}\right]^{\mathrm{T}}\left[\sum_{j \in N(i)} e_{i j}\right] \\
& +\frac{c}{2} \cdot \sum_{i=1}^{N}\left[\sum_{j \in N(i)} e_{i j}(t-\tau(t))\right]^{\mathrm{T}}\left[\sum_{j \in N(i)} e_{i j}(t-\tau(t))\right] \\
= & \frac{c}{2} \lambda_{\max }\left(\Gamma \Gamma^{\mathrm{T}}\right) \cdot\left[x^{\mathrm{T}}\left(L^{2} \otimes I_{n}\right) x\right]+\frac{c}{2} \cdot\left[x^{\mathrm{T}}(t-\tau(t))\left(L^{2} \otimes I_{n}\right) x(t-\tau(t))\right]
\end{aligned}
$$

Besides, by using Assumptions 2 and 3, (25), and (26), one obtains that

$$
\begin{aligned}
& -\sum_{i=1}^{N}\left[\sum_{j \in N(i)} e_{i j}(t-\tau(t))\right]^{\mathrm{T}} P\left[\sum_{j \in N(i)} e_{i j}(t-\tau(t))\right] \cdot(1-\dot{\tau}(t)) \\
& =-(1-\dot{\tau}(t)) \cdot x^{\mathrm{T}}(t-\tau(t))\left(P L^{2} \otimes I_{n}\right) x(t-\tau(t)) \\
& \leq-(1-\bar{\tau}) \cdot x^{\mathrm{T}}(t-\tau(t))\left(P L^{2} \otimes I_{n}\right) x(t-\tau(t))
\end{aligned}
$$


and

$$
\begin{aligned}
& \frac{1}{4} \sum_{i=1}^{N} \sum_{j \in N(i)} \tilde{\sigma}^{\mathrm{T}}\left(t, e_{i j}(t), e_{i j}(t-\tau(t))\right) \tilde{\sigma}\left(t, e_{i j}(t), e_{i j}(t-\tau(t))\right) \\
& \leq \frac{1}{4} \sum_{i=1}^{N} \sum_{j \in N(i)}\left[\gamma_{1} e_{i j}^{\mathrm{T}}(t) e_{i j}(t)+\gamma_{2} e_{i j}^{\mathrm{T}}(t-\tau(t)) e_{i j}(t-\tau(t))\right] \\
& =\frac{1}{2} \gamma_{1} \cdot \frac{1}{2} \sum_{i=1}^{N} \sum_{j \in N(i)} e_{i j}^{\mathrm{T}}(t) e_{i j}(t)+\frac{1}{2} \gamma_{2} \cdot \frac{1}{2} \sum_{i=1}^{N} \sum_{j \in N(i)} e_{i j}^{\mathrm{T}}(t-\tau(t)) e_{i j}(t-\tau(t)) \\
& =\frac{1}{2} \gamma_{1} \cdot x^{\mathrm{T}}\left(L \otimes I_{n}\right) x+\frac{1}{2} \gamma_{2} \cdot x^{\mathrm{T}}(t-\tau(t))\left(L \otimes I_{n}\right) x(t-\tau(t))
\end{aligned}
$$

Combining the above results, and substitute (26) - (31) into (24), we have

$$
\begin{aligned}
E & \propto \\
& +\frac{1}{2} \beta_{2} \cdot x^{\mathrm{T}}(t-\tau(t))\left(L \otimes I_{n}\right) x(t-\tau(t))+\frac{c}{2} \lambda_{\max }\left(\Gamma \Gamma^{\mathrm{T}}\right) \cdot\left[x^{\mathrm{T}}\left(L^{2} \otimes I_{n}\right) x\right] \\
& +\frac{c}{2} \cdot\left[x^{\mathrm{T}}(t-\tau(t))\left(L^{2} \otimes I_{n}\right) x(t-\tau(t))\right]-\rho b x^{\mathrm{T}}\left(L^{2} \otimes \Gamma\right) x \\
& +x^{\mathrm{T}}\left(P L^{2} \otimes I_{n}\right) x-(1-\bar{\tau}) \cdot x^{\mathrm{T}}(t-\tau(t))\left(P L^{2} \otimes I_{n}\right) x(t-\tau(t)) \\
& \left.+\frac{1}{2} \gamma_{1} \cdot x^{\mathrm{T}}\left(L \otimes I_{n}\right) x+\frac{1}{2} \gamma_{2} \cdot x^{\mathrm{T}}(t-\tau(t))\left(L \otimes I_{n}\right) x(t-\tau(t))\right\} \\
= & E\left\{x ^ { \mathrm { T } } \left[\left(\sqrt{\lambda_{\max }\left(A^{\mathrm{T}} A\right)} I_{N}+\frac{1}{2}\left(\beta_{1}+1\right) I_{N}+\frac{1}{2} \gamma_{1} I_{N}\right.\right.\right. \\
& \left.\left.+\frac{c}{2} \lambda_{\max }\left(\Gamma \Gamma^{\mathrm{T}}\right) L+P L\right) L \otimes I_{n}\right] x+x^{\mathrm{T}}[(-\rho b L) L \otimes \Gamma] x \\
& \left.+x^{\mathrm{T}}(t-\tau(t))\left[\left(\frac{1}{2} \beta_{2} I_{N}+\frac{1}{2} \gamma_{2} I_{N}+\frac{c}{2} L-P(1-\bar{\tau}) L\right) L \otimes I_{n}\right] x(t-\tau(t))\right\}
\end{aligned}
$$

Let $\eta=\min \left\{\eta_{1}, \eta_{2}, \cdots, \eta_{n}\right\}$, then we have

$$
\begin{array}{rl}
E & \mathscr{L} V(t) \leq E\left\{x ^ { \mathrm { T } } \left[\left(\sqrt{\lambda_{\max }\left(A^{\mathrm{T}} A\right)} I_{N}+\frac{1}{2}\left(\beta_{1}+1\right) I_{N}+\frac{1}{2} \gamma_{1} I_{N}\right.\right.\right. \\
& \left.\left.+\frac{c}{2} \lambda_{\max }\left(\Gamma \Gamma^{\mathrm{T}}\right) L+P L\right) L \otimes I_{n}\right] x+x^{\mathrm{T}}\left[(-\rho b \eta L) L \otimes I_{n}\right] x \\
& \left.+x^{\mathrm{T}}(t-\tau(t))\left[\left(\frac{1}{2} \beta_{2} I_{N}+\frac{1}{2} \gamma_{2} I_{N}+\frac{c}{2} L-P(1-\bar{\tau}) L\right) L \otimes I_{n}\right] x(t-\tau(t))\right\} \\
= & E\left\{x ^ { \mathrm { T } } \left[\left(\sqrt{\lambda_{\max }\left(A^{\mathrm{T}} A\right)} I_{N}+\frac{1}{2}\left(\beta_{1}+1\right) I_{N}+\frac{1}{2} \gamma_{1} I_{N}\right.\right.\right. \\
& \left.\left.+\frac{c}{2} \lambda_{\max }\left(\Gamma \Gamma^{\mathrm{T}}\right) L+P L-\rho b \eta L\right) L \otimes I_{n}\right] x \\
& \left.+x^{\mathrm{T}}(t-\tau(t))\left[\left(\frac{1}{2} \beta_{2} I_{N}+\frac{1}{2} \gamma_{2} I_{N}+\frac{c}{2} L-P(1-\bar{\tau}) L\right) L \otimes I_{n}\right] x(t-\tau(t))\right\} \\
= & E\left\{-x^{\mathrm{T}}(t) \prod_{1}\left(L \otimes I_{n}\right) x(t)+x^{\mathrm{T}}(t-\tau(t)) \Pi_{2}\left(L \otimes I_{n}\right) x(t-\tau(t))\right\}
\end{array}
$$

where 


$$
\begin{gathered}
\prod_{1}=\rho b \eta L-\sqrt{\lambda_{\max }\left(A^{\mathrm{T}} A\right)} I_{N}-\frac{1}{2}\left(\beta_{1}+1\right) I_{N}-\frac{1}{2} \gamma_{1} I_{N}-\frac{c}{2} \lambda_{\max }\left(\Gamma \Gamma^{\mathrm{T}}\right) L-P L \\
\prod_{2}=\frac{1}{2} \beta_{2} I_{N}+\frac{1}{2} \gamma_{2} I_{N}+\frac{c}{2} L-P(1-\bar{\tau}) L
\end{gathered}
$$

Note that condition (16) in Theorem 1 yields

$$
\left[\frac{1}{2}\left(\beta_{1}+\beta_{2}+\gamma_{1}+\gamma_{2}+1\right)+\sqrt{\lambda_{\max }\left(A^{\mathrm{T}} A\right)}\right] I_{N}<\left[\rho b \eta-\frac{c}{2}-P \bar{\tau}-\frac{c}{2} \lambda_{\max }\left(\Gamma \Gamma^{\mathrm{T}}\right)\right] L
$$

we obtain

$$
\begin{aligned}
\prod_{2}-\prod_{1}= & {\left[\frac{1}{2}\left(\beta_{1}+\beta_{2}+\gamma_{1}+\gamma_{2}+1\right)+\sqrt{\lambda_{\max }\left(A^{\mathrm{T}} A\right)}\right] I_{N} } \\
& -\left[\rho b \eta-\frac{c}{2}-P \bar{\tau}-\frac{c}{2} \lambda_{\max }\left(\Gamma \Gamma^{\mathrm{T}}\right)\right] L \\
< & 0
\end{aligned}
$$

Namely, $\Pi_{2}<\Pi_{1}$. Therefore, from the LaSalle invariance principle [27] of stochastic differential equations with time-varying delays, the distributed synchronization of the stochastic complex network with time-varying delays in (3) via randomly occurring control and updating law can be achieved in mean square. This concludes the proof.

When the delay in the stochastic complex network in (3) is a constant delay, the following Corollary 1 can be obtained.

Corollary 1: Suppose that the nonlinear function $f(\cdot, \cdot, \cdot)$ in the stochastic complex network (3) satisfies Assumption 1, and the noise intensity function $\sigma(\cdot, \cdot, \cdot)$ satisfies Assumption 2, then the stochastic complex network (3) will achieve synchronization in mean square under the distributed adaptive controller (4) and updating law (7), if

$$
\left[\frac{1}{2}\left(\beta_{1}+\beta_{2}+\gamma_{1}+\gamma_{2}+1\right)+\sqrt{\lambda_{\max }\left(A^{\mathrm{T}} A\right)}\right] I_{N}<\left[\rho b \eta-\frac{c}{2}-\frac{c}{2} \lambda_{\max }\left(\Gamma \Gamma^{\mathrm{T}}\right)\right] L
$$

Proof: The Lyapunov function constructed at this time becomes

$$
\begin{aligned}
V(t)= & \frac{1}{4} \sum_{i=1}^{N} \sum_{j \in N(i)} e_{i j}^{\mathrm{T}} e_{i j}+\frac{\rho}{2 \xi \alpha} \sum_{i=1}^{N}\left(\varepsilon_{i}(t)-b\right)^{2} \\
& +\sum_{i=1}^{N} \int_{t-\tau}^{t}\left[\sum_{j \in N(i)} e_{i j}(s)\right]^{\mathrm{T}} P\left[\sum_{j \in N(i)} e_{i j}(s)\right] \mathrm{d} s
\end{aligned}
$$

where $b$ is a positive constant. The rest of the proof is similar to the proof of Theorem 1, which is omitted here.

When the stochastic complex network (3) does not contain time delay, the following simpler Corollary 2 can be obtained.

Assumption 4 [9]: The nonlinear function $f(\cdot, \cdot): \mathbb{R} \times \mathbb{R}^{n} \rightarrow \mathbb{R}^{n}$ is assumed to satisfy a Lipschitz condition, that is, there exists a constant $\beta>0$, such that the following inequality holds for all $\xi_{1}(t), \xi_{2}(t) \in \mathbb{R}^{n}$ :

$$
\left\|f\left(t, \xi_{1}(t)\right)-f\left(t, \xi_{2}(t)\right)\right\|^{2} \leq \beta\left\|\xi_{1}(t)-\xi_{2}(t)\right\|^{2}
$$

Assumption 5 [26]: Assume that the noise intensity function 
$\sigma(\cdot, \cdot): \mathbb{R} \times \mathbb{R}^{n} \rightarrow \mathbb{R}^{n}$ is uniformly Lipschitz continuous with respect to $t$ if there exist a constant $\gamma>0$, such that the following inequality holds for all $\xi_{1}, \xi_{2} \in \mathbb{R}^{n}:$

$$
\operatorname{trace}\left[\left(\sigma\left(t, \xi_{1}\right)-\sigma\left(t, \xi_{2}\right)\right)^{\mathrm{T}}\left(\sigma\left(t, \xi_{1}\right)-\sigma\left(t, \xi_{2}\right)\right)\right] \leq \gamma\left(\xi_{1}-\xi_{2}\right)^{\mathrm{T}}\left(\xi_{1}-\xi_{2}\right)
$$

Corollary 2: Suppose that Assumptions 4 and 5 hold, then the stochastic complex network (3) will achieve synchronization in mean square under the distributed adaptive controller (4) and updating law (7), if

$$
\left[\frac{1}{2}(\beta+\gamma+1)+\sqrt{\lambda_{\max }\left(A^{\mathrm{T}} A\right)}\right] I_{N}<(\rho b \eta+c) L
$$

Proof: The Lyapunov function constructed at this time becomes

$$
V(t)=\frac{1}{4} \sum_{i=1}^{N} \sum_{j \in N(i)} e_{i j}^{\mathrm{T}} e_{i j}+\frac{\rho}{2 \xi \alpha} \sum_{i=1}^{N}\left(\varepsilon_{i}(t)-b\right)^{2}
$$

where $b$ is a positive constant. The rest of the proof is similar to the proof of Theorem 1, and will not be detailed here.

\section{Conclusion}

In this paper, synchronization criteria were investigated for the stochastic complex networks with time-varying delays and random noise via randomly occurring control and updating law. We use two Bernoulli random variables to describe the occurrence of distributed adaptive control, and update it according to a certain probability. The adaptive control and update rules connected to the network node are only related to the state information of itself and its neighbor nodes, and distributed synchronization is realized through the feedback of the state information of neighbor nodes. Based on Lyapunov stability theory, LaSalle invariance principle, combined with the use of the properties of matrix Kronecker product, stochastic differential equation theory and other related tools, by constructing the appropriate Lyapunov functional, the sufficient conditions for the distributed synchronization of such stochastic complex networks in mean square are obtained.

\section{Acknowledgements}

This work was supported by the Fujian Provincial Department of Education Young and Middle-aged Teacher Education Research Project (JAT201031). The author would also like to thank the Editor-in-Chief, the Associate Editor, and the anonymous reviewers for their careful reading of the manuscript and constructive comments.

\section{Conflicts of Interest}

The authors declare no conflicts of interest regarding the publication of this paper.

\section{References}

[1] Strogatz, S.H. (2004) Sync: How Order Emerges from Chaos in the Universe, Na- 
ture, and Daily Life. Hyperion, New York.

[2] Ott, E. (2002) Chaos in Dynamical Systems. 2nd Edition, Cambridge University Press, Cambridge. https://doi.org/10.1017/CBO9780511803260

[3] Tang, Y., Gao, H., Zou, W. and Kurths, J. (2012) Identifying Controlling Nodes in Neuronal Networks in Different Scales. PLoS ONE, 7, e41375. https://doi.org/10.1371/journal.pone.0041375

[4] Zhou, L. and Tan, F. (2019) A Chaotic Secure Communication Scheme Based on Synchronization of Double-Layered and Multiple Complex Networks. Nonlinear Dynamics, 96, 869-883. https://doi.org/10.1007/s11071-019-04828-7

[5] Liang, J., Wang, Z. and Liu, X. (2009) State Estimation for Coupled Uncertain Stochastic Networks with Missing Measurements and Time-Varying Delays: The Discrete-Time Case. IEEE Transactions on Neural Networks, 20, 781-793. https://doi.org/10.1109/TNN.2009.2013240

[6] Ma, L., Wang, Z., Hu, J., Bo, Y. and Zhi, G. (2010) Robust Variance-Constrained Filtering for a Class of Nonlinear Stochastic Systems with Missing Measurements. Signal Processing, 90, 2060-2071. https://doi.org/10.1016/j.sigpro.2010.01.010

[7] Gao, H.J., Meng, X.Y. and Chen, T.W. (2010) Stabilization of Networked Control Systems via Dynamic Output-Feedback Controllers. SIAM Journal on Control and Optimization, 48, 3643-3658. https://doi.org/10.1137/070679132

[8] Hunt, D., Korniss, G. and Szymanski, B. (2010) Network Synchronization in a Noisy Environment with Time Delays: Fundamental Limits and Trade-Offs. Physical Review Letters, 105, Article ID: 068701. https://doi.org/10.1103/PhysRevLett.105.068701

[9] Wang, Z., Wang, Y. and Liu, Y. (2010) Global Synchronization for Discrete-Time Stochastic Complex Networks with Randomly Occurred Nonlinearities and Mixed Time-Delays. IEEE Transactions on Neural Networks, 21, 11-25. https://doi.org/10.1109/TNN.2009.2033599

[10] Guo, L. (2009) Control and Synchronization of Complex Networks with stochastic and Uncertain Factors. Thesis, Huazhong University of Science and Technology, Wuhan.

[11] Bei, Z., Zhuang, J., Liu, H., Cao, J. and Xia, Y. (2018) Master-Slave Synchronization of a Class of Fractional-Order Takagi-Sugeno Fuzzy Neural Networks. Advances in Difference Equations, 2018, Article No. 473. https://doi.org/10.1186/s13662-018-1918-y

[12] Tang, Y. and Wong, W.K. (2013) Distributed Synchronization of Coupled Neural Networks via Randomly Occurring Control. IEEE Transactions on Neural Networks and Learning Systems, 24, 435-447. https://doi.org/10.1109/TNNLS.2012.2236355

[13] Liu, X. (2020) Distributed Synchronization of Coupled Time-Delay Neural Networks Based on Randomly Occurring Control. Applied Mathematics, 11, 698-711. https://doi.org/10.4236/am.2020.117047

[14] Yang, X., Lu, J., Ho, D. and Song, Q. (2018) Synchronization of Uncertain Hybrid Switching and Impulsive Complex Networks. Applied Mathematical Modelling, 59, 379-392. https://doi.org/10.1016/j.apm.2018.01.046

[15] Song, Y. and Jian, X. (2012) Inphase and Antiphase Synchronization in a DelayCoupled System with Applications to a Delay-Coupled Fitzhugh-Nagumo System. IEEE Transactions on Neural Networks \& Learning Systems, 23, 1659-1670. https://doi.org/10.1109/TNNLS.2012.2209459

[16] Liu, X., Su, H. and Chen, M.Z. (2016) A Switching Approach to Designing Finite- 
Time Synchronization Controllers of Coupled Neural Networks. IEEE Transactions on Neural Networks \& Learning Systems, 27, 471-482. https://doi.org/10.1109/TNNLS.2015.2448549

[17] Guo, B., Wu, Y., Xiao, Y. and Zhang, C. (2018) Graph-Theoretic Approach to Synchronizing Stochastic Coupled Systems with Time-Varying Delays on Networks via Periodically Intermittent Control. Applied Mathematics and Computation, 331, 341-357. https://doi.org/10.1016/j.amc.2018.03.020

[18] Li, Y., Lou, J., Wang, Z. and Alsaadi, F.E. (2018) Synchronization of Dynamical Networks with Nonlinearly Coupling Function under Hybrid Pinning Impulsive Controllers. Journal of the Franklin Institute, 355, 6520-6530. https://doi.org/10.1016/j.jfranklin.2018.06.021

[19] Hespanha, J., Naghshtabrizi, P. and Xu, Y. (2007) A Survey of Recent Results in Networked Control Systems. Proceedings of the IEEE, 95, 138-162. https://doi.org/10.1109/JPROC.2006.887288

[20] Sun, X., Liu, G., Rees, D. and Wang, W. (2008) Stability of Systems with Controller Failure and Time-Varying Delay. IEEE Transactions on Automatic Control, 53, 2391-2396. https://doi.org/10.1109/TAC.2008.2007528

[21] Mao, X. (2007) Stochastic Differential Equations and Applications. 2nd Edition, Horwood, Chichester.

[22] Dai, A.D. (2014) Synchronization Analysis and Control for Switching Complex Networks Based on M-Matrix Approach. Ph.D. Thesis, DongHua University, Shanghai.

[23] Sun, D.W. (2017) Research on Quantitative Feedback and Event-Triggering Control of Networked Control Systems. Master Thesis, Dalian Maritime University, Dalian.

[24] Yang, X. and Cao, J. (2009) Stochastic Synchronization of Coupled Neural Networks with Intermittent Control. Physics Letters A, 373, 3259-3272.

https://doi.org/10.1016/j.physleta.2009.07.013

[25] Shi, L., Yang, X., Li, Y. and Feng, Z. (2016) Finite-Time Synchronization of Nonidentical Chaotic Systems with Multiple Time-Varying Delays and Bounded Perturbations. Nonlinear Dynamics, 83, 75-87. https://doi.org/10.1007/s11071-015-2310-z

[26] Zhu, Q. and Cao, J. (2011) Adaptive Synchronization under almost Every Initial Data for Stochastic Neural Networks with Time-Varying Delays and Distributed Delays. Communications in Nonlinear Science and Numerical Simulation, 16, 2139 2159. https://doi.org/10.1016/j.cnsns.2010.08.037

[27] Mao, X. (2002) A Note on the Lasalle-Type Theorems for Stochastic Differential Delay Equations. Journal of Mathematical Analysis and Applications, 268, 125-142. https://doi.org/10.1006/jmaa.2001.7803 